\title{
Impact for Timely and Reliable Data Transmission in Industrial Areas- A Review
}

\author{
Atiq Ali Khan ${ }^{a}$, Dr. Anil Kumar ${ }^{\text {b }}$ \\ a Research Scholar, Dept. of Electronics and Communication Engineering, \\ Sri Satya Sai University of Technology \& Medical Sciences, Sehore, Bhopal Indore Road, Madhya Pradesh, India \\ ${ }^{\mathbf{b}}$ Research Guide, Dept. of Electronics and Communication Engineering, \\ Sri Satya Sai University of Technology \& Medical Sciences, Sehore, Bhopal Indore Road, Madhya Pradesh, India
}

Article History: Received: 11 January 2021; Accepted: 27 February 2021; Published online: 5 April 2021

\begin{abstract}
:
By breaking down the impact for timely and reliable data transmission in industrial areas, the authors uncover that couple of distributions shed light on this viewpoint. By setting up these discoveries, this paper gives a stage to the comprehension of what might actually shape keen plan designing and its plan related exercises. To achieve this, the authors lead a methodical review of the literature, which gives different discoveries. As the new impact plan to an enormous degree characterizes the worth chain for an organization, the test lies in guaranteeing that the planned item will assist the organization with profiting the Industrial Areas. These discoveries are then dissected by 12 experts both from the scholarly community and the business, and the discoveries' relations to the impact for timely and reliable data transmission in industrial areas and their impact on the item advancement measure is examined. Industrial Areas are generally connected with what changes in regards to the operations of an undertaking mean for said venture's assembling frameworks. In any case, the impact of these changes surpasses the creation frameworks themselves; rather, they influence the whole worth chain, from the item plan and advancement measure through assembling and store network the board to promoting and removal. Therefore, the reason for this investigation is to set up highlights and properties that will shape all through the industrial areas and into a savvy configuration designing.
\end{abstract}

Keywords: Data, Transmission, Industrial, Impact, Timely, Reliable

\section{Introduction}

The utilization of data transmission offices speeds up the assortment and scattering of data and grants endorsers with economical terminal gear to appreciate the administrations of huge PC habitats. It started growing widely in numerous different nations also. Data. A data transmission framework was placed into operation in the Onega programmed control framework for checking cash settlements. In this framework, data are communicated along broadcast and phone channels at speeds of 50 and 600 pieces/sec, individually. Data transmission was subsequently joined in the Pagoda framework for gathering meteorological data and in numerous programmed control frameworks in industry and the state administration. Association of the National Data Transmission System, which will ultimately give data transmission administrations to all services and divisions, the main phase of the framework to be set up is a message type network that communicates data at paces of up to 200 pieces/sec.

As of now, data transmission is perhaps the most quickly creating areas of innovation. There were close to 1,000 data transmission terminal units in the whole world. The figure rose to 35, 000 and 150,000. The number was relied upon to surpass 1 million. In numerous nations the normal yearly development has been 70-100\%. Data are communicated in numerous nations fundamentally through exchanged message or phone networks. Since these networks are planned essentially for communicating wires or phone messages, uncommon terminal gadgets are added for data transmission. The utilization of mistake amending codes makes conceivable the location of most blunders, and the mistakes are typically adjusted by a consequently rehashed transmission.

The DTD is connected with the PC gear through an intermediate data transporter, which generally is punched tape, or through electric circuit. An illustration of the subsequent sort is the universally useful gear of the United System of Computers of the communist nations. The expression "data transmission" is additionally applied to the genuine cycle of sending the data. Data transmission is in a developmental stage and is creating in the accompanying primary ways: the formation of extraordinary data transmission networks that include both the advancement of exchanging focuses offering improved support to endorsers and the presentation of computerized correspondences channels shaped by frameworks with time-division line multiplexing; the ideal blend of the improvement of new networks with the utilization of existing phone and transmit networks; the increment of the proficiency of high-rate correspondence channels including the accomplishment of transmission paces of 4,800 pieces/sec or more along phone channels; the rearrangements of the DTD for low-rate correspondence; and the expansion of the exactness and dependability of interchanges. With the finish of data transmission, they get back to the underlying mode. On account of phone channels, for instance, it utilizes recurrence, stage, and other more unpredictable kinds of tweak, just as different types of sign coding and recoding. 

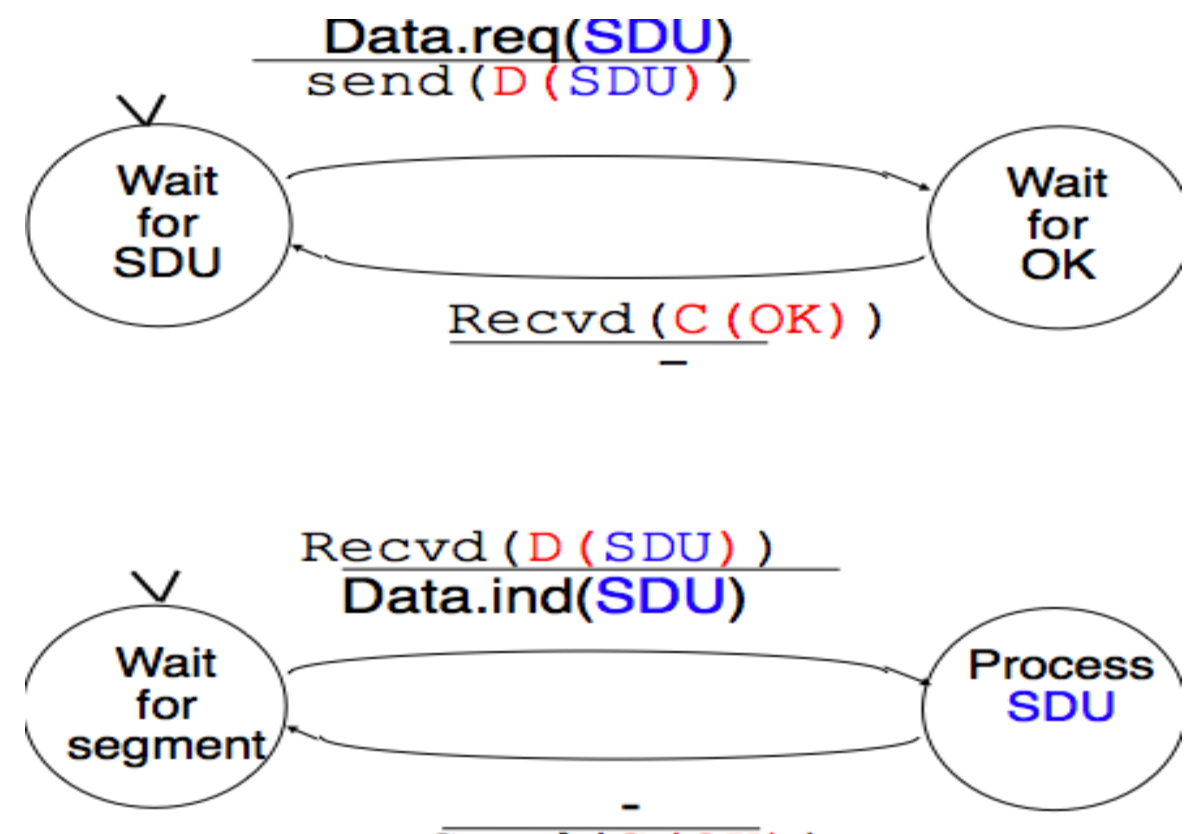

\section{Send (C(OK) )}

Figure 1 Data Transmission

To send enormous volumes of data for instance, between two PC places-no exchanged (immediate, rented) correspondences channels are utilized; data can be sent at paces of 2,400 pieces/sec or more with non-exchanged phone channels. Channels have given data transmission a likelihood of blunder of 10-3-10-5; by methods for mistake control gadgets the likelihood can be decreased to 10-6-10-8. Two standards of exchanging are being used: channel exchanging, where a through channel from endorser of supporter is coordinated for the hour of correspondence, and message exchanging. The last sort of DTD grants supporters of "discuss" straightforwardly with a PC whose product has a bunch of projects that control the arrangement of distant data handling (the trading of data with endorser terminals and with different PCs). Instances of the principal sort of DTD are the Akkord-50 normalized DTD, which is utilized in the USSR with broadcast channels and has a pace of up to 50 pieces/sec, and the Akkord-1200, which is utilized with phone channels and has paces of 600 or 1,200 pieces/sec. With data input-yield by an intermediate transporter, with electrical data input-yield; images: inputyield gadgets, punched tape, blunder control gadget, signal change gadget, broadcast or phone unit, switch, supporter line, exchanging focus, data transmission multiplexer, control unit, data transmission gadget endorser's DTD changes over data signals into a structure appropriate for transmission along the correspondence channel. An exchanged association is made "physically" by methods for the TU. Where essential, the DTD incorporates a gadget to secure data against mistakes that emerge in the interchanges channel in view of commotion.

The DTD may likewise contain helper units, for example, calling and talk units and checking gadgets. Mistake discovery should likewise be possible by noncore techniques using a quality identifier, which dissects the known boundaries of the sign, for example, abundancy, recurrence, and length. Data transmission every now and again requires more noteworthy dependability, rate, and precision of transmission as a result of the more prominent significance of the data being sent and the inconceivability of intelligent checking by people during the sending and getting measures. They can offer higher quality support to endorsers as far as exactness and pace of transmission, the chance of picking the class of work need and speed, and the chance of multi address correspondence, and they offer extra administrations too. Phone networks are utilized to communicate computerized data as well as simple data, or data in a constant structure, for instance, cardiograms.

The close down is done in the typical manner by methods for the TU. In the event that the endorser has satisfactory insurance against mistakes in his PC hardware, other security isn't given in the DTD. Such data is called data. The main contrast between data transmission and broadcast, phone, and different sorts of correspondence is that the data, or data, is sent or gotten by a machine as opposed to a person; in data transmission from PC to PC there is no individual on one or the flip side of the correspondence line. Phone and broadcast networks can't fulfill the best requests made of data transmission. Toward the finish of their discussion, or subsequent to trading broadcast messages, the members consent to change to the data transmission mode, and they associate the interchanges channel to the DTD. The part of broadcast communications worried about the transmission of data addressed, based on foreordained principles, in a formalized structure by images or simple signals; the data either is expected for machine preparing(for instance, by PCs) or has just gone through machine preparing. The association can likewise be made consequently, with PC control. This sort of DTD has no info yield gadgets. Notwithstanding the standard message or phone unit (TU), a data transmission gadget (DTD), which makes the PC gear viable with the correspondences channel, and an interchanges channel 
switch (S) are introduced at the endorser's terminal. The association of the DTD to an exchanged phone or broadcast network is convenient for little volumes of communicated data, when the complete time the supporter line will be utilized for discussion and data transmission won't surpass 6-12 minutes now and again of pinnacle load. In the last case, the message is communicated in full from the sender-supporter of the closest exchanging focus, where it is incidentally put away; when a divert in the essential (appointed) bearing turns out to be free, the message is sent on in stages, from focus to focus, until it is gotten by the recipient endorser. PCs are being utilized increasingly more much of the time to control exchanging at the focuses. Starting with the last part of the 1960 's, in this manner, extraordinary exchanged data transmission networks have come into utilization. Along with PC innovation, data transmission fills in as the specialized base for data and figuring frameworks, including programmed control frameworks of different degrees of multifaceted nature.

\section{Literature Review}

Test Md Abul Kalam Azad (2020): In planning an ongoing MAC convention for wireless sensor networks, it is attractive to abbreviate the data procurement process duration to fulfill more applications with a more tight time imperative in data assortment [1]. To accomplish this, one frequently attempts to improve throughput by utilizing an opening reuse method. Then, the opened sense numerous access convention that utilizes a sharable space was demonstrated to be profitable for timely and reliable data delivery [1]. Notwithstanding, since the hubs at lower tree levels measure more packets or bigger packets with data total, they need a bigger sharable opening. In this way, it is difficult to utilize an opening reuse procedure because of the size variety of sharable spaces [1]. To handle this issue, we attempt to diminish the size of an amassed packet by restricting the quantity of packets for collection utilizing two channels [1]. On the off chance that each hub is permitted to send a solitary packet during data obtaining process duration, an equivalent estimated sharable opening can be designated to each tree level [1]. It is demonstrated that the proposed approach can lessen data procurement process duration altogether while keeping up great unwavering quality in data transmission contrasted and opened sense numerous access and furthermore diminish energy utilization significantly contrasted and another multi-channel MAC convention. Shakyawar (2019): 'Big data' is huge measures of data that can do some incredible things [2]. It has become a subject of uncommon interest for as far back as twenty years in view of an incredible potential that is covered up in it [2]. Different public and private area ventures create, store, and investigate big data with intend to improve the administrations they give [2]. In the medical care industry, different hotspots for big data incorporate emergency clinic records, clinical records of patients and consequences of clinical assessments, and gadgets that are a piece of internet of things [2]. Biomedical exploration likewise produces a critical bit of big data applicable to public medical services. This data requires appropriate administration and investigation to determine important data. Something else, looking for arrangement by investigating big data rapidly gets tantamount to finding a needle in the bundle [2]. There are different difficulties related with each progression of taking care of big data which must be outperformed by utilizing high-end registering answers for big data examination [2]. That is the reason, to give important answers for improving general wellbeing, medical services suppliers are needed to be completely outfitted with suitable framework to efficiently produce and examine big data. An effective administration, investigation, and understanding of big data can change the game by opening new roads for current medical care [2]. That is actually why different ventures, including the medical care industry, are finding a way to change over this potential into better administrations and monetary focal points. With a solid integration of biomedical and medical care data, current medical services associations can alter the clinical treatments and customized medication.

Sinha(2019): Disaster the executives expects to moderate the likely harm from the catastrophes, guarantee immediate and reasonable help to the people in question, and achieve compelling and quick recuperation [3]. These goals require an arranged and powerful salvage operation post such fiascos. Various sorts of data about the impact of the calamity are, subsequently, needed for arranging a compelling and immediate help operation [3]. The IoT innovation accessible today is very adult and can possibly be valuable in misfortune circumstances [3]. This paper investigates the necessities for arranging salvage operation for such cataclysmic events and proposes an IoT based answer for provide food the distinguished prerequisites. The proposed arrangement is additionally approved utilizing the assignment innovation fit (TTF) approach for examining the meaning of the appropriation of IoT innovation for fiasco the board. Results from the exploratory examination set up the center components of the errand necessities and the TTF develops [3]. Results from the corroborative factor investigation utilizing PLS way displaying, further, recommend that both undertaking prerequisites and IoT innovation altogether affect the IoT TTF in the debacle the board situation [3]. This paper makes huge commitments in the improvement of suitable develops for displaying TTF for IoT Technology with regards to fiasco the executives.

Yamila M.Omar (2019): The assembling area is feeling the squeeze to build benefit in a growingly serious global market in which separation isn't attached to fabricated items or used advancements however to business measures streamlining [4]. In this specific circumstance, business analytics offer the chance to outfit the information and worth covered up inside big business data frameworks to reform advancement, upgrade store network the board and creation, precisely target advertising and deals endeavors, just as create and oversee 
productive after-deals administrations [4]. While the literature to date presents various explicit applications in which business analytics procedures were effectively conveyed to improve explicit specialty units, it is apparent that an extensive endeavor approach is absent [4]. In the current work, a pathway to achieve market administration through the powerful utilization of business analytics is characterized recommending center should revolve around three progressively testing hindrances [4]. Initially, "normalization" of assortment, total and capacity of data should be cultivated. At that point, an "authoritative culture development" that grows out of instinct and accepts data-driven dynamic is expected to make the ideal environment for business analytics to deliver noteworthy outcomes and suggestions [4]. Thus, these should manage "plan of action advancement" endeavors to handle new esteem creation, and catch and secure market initiative.

Xue-Ming Yuan (2019): With advancement of Industry 4.0, how could we work our creation lines and manufacturing plants, how might we oversee and improve stock, how could we convey our laborers, how might we maintain our organizations, how might we deal with our inventory chains? This section expects to highlight the impact of Industry 4.0 on assembling frameworks and administrations, just as supply chains, specifically, on stock frameworks and improvement [5]. An integrative R\&D structure for stock frameworks demonstrating and streamlining is proposed, which coordinates our R\&D exertion in displaying and advancing stock frameworks with Industry 4.0 [5]. The world is encountering Industry 4.0, the fourth industrial transformation [5]. The primary industrial transformation occurred in the eighteenth century with the presentation of mechanical creation machines fueled by water and steam [5]. The second industrial upheaval began toward the start of the 20th century with large scale manufacturing fueled by electric energy. The third industrial unrest came in 1970s with creation computerization utilizing hardware, PCs and data innovation [5]. The current industrial insurgency started in the right on time of this thousand years with self-sufficient creation utilizing Cyber-Physical Systems (CPS), Internet of Things (IoT) and Internet of Services (IoS) [5]. This digitization not just empowers the integration of cycles and frameworks across organizations and industrial areas, yet in addition makes new plans of action and worth generation openings [5].

Pradittasnee (2017): Reliable data transmissions are trying in industrial wireless sensor networks as channel conditions change over the long run. Quick changes in channel conditions require exact assessment of the steering way execution and timely update of the directing data [6]. Be that as it may, this isn't very much satisfied in existing directing methodologies [6]. Tending to this issue, this paper presents joined worldwide and neighborhood update measures for proficient course update and support, and fuses them with a progressive proactive directing system [6]. While the worldwide interaction refreshes the steering way with a generally extensive stretch, the nearby cycle with a more limited period checks potential directing way issues [6]. A hypothetical demonstrating is created to depict the cycles [6]. Through reenactments, the introduced approach is appeared to decrease start to finish delay up to multiple times for enormous networks, while improving packet reception ratio (PRR) in correlation with progressive and proactive directing conventions ROL/NDC, DSDV, and DSDV with IPv6 Routing Protocol for Low-Power and Lossy Networks' Trickle calculation [6]. Contrasted and receptive directing conventions AODV and Ad Hoc On-request Multipath Distance Vector, it gives comparative PRR while decreasing start to finish delay more than 15 times [6].

L. Pradittasne (2017): Big Data (BD), with their capability to learn esteemed experiences for upgraded dynamic cycle, have as of late pulled in generous premium from the two scholastics and experts [7]. Big Data Analytics (BDA) is progressively turning into a moving practice that numerous associations are receiving to build important data from BD [7]. The analytics cycle, including the arrangement and utilization of BDA devices, is seen by associations as an apparatus to improve operational effectiveness however it has vital potential, drive new income streams and gain upper hands over business rivals [7]. Notwithstanding, there are various kinds of scientific applications to consider. Accordingly, preceding rushed use and purchasing expensive BD devices, there is a requirement for associations to initially comprehend the BDA scene [7]. Given the critical idea of the $\mathrm{BD}$ and BDA, this paper presents a cutting edge review that presents a comprehensive perspective on the BD difficulties and BDA strategies guessed/proposed/utilized by associations to help other people comprehend this scene with the target of settling on hearty venture choices [7]. In doing as such, deliberately investigating and integrating the surviving examination distributed on BD and BDA territory [7]. All the more explicitly, the authors look to answer the accompanying two chief inquiries: Q1 what are the various sorts of BD challenges estimated/proposed/faced by associations? Also, Q2what are the various kinds of BDA techniques guessed/proposed/utilized to beat BD challenges? This precise literature review (SLR) is helped out through noticing and understanding the previous patterns and surviving examples/subjects in the BDA research territory, assessing commitments, summing up information, along these lines distinguishing restrictions, suggestions and potential further examination roads to help the scholastic local area in investigating research topics/designs [7]. Along these lines, to follow the usage of BD methodologies, a profiling strategy is utilized to dissect articles (distributed in English-talking peer-reviewed diaries somewhere in the range of 1996 and 2015) removed from the Scopus database [7]. The examination introduced in this paper has recognized applicable BD research considers that have contributed both adroitly and experimentally to the extension and gathering of scholarly abundance to the BDA in innovation and hierarchical asset the board discipline. 
Burritt, R (2016): The paper tries to analyze how, through ecological bookkeeping, the more extensive setting of corporate supportability could be joined into the creating vision for Industry 4.0, the fourth industrial unrest [8]. A contention is built up that through commitment of the calling ecological bookkeeping could be improved and could piggy-back at minimal additional expense on huge genuine and potential ventures being made in Industry 4.0 foundation intended for digitization of business. Industry 4.0 could be utilized effectively as a premise whereupon to use both outside ecological bookkeeping and natural administration bookkeeping [8]. Another scholarly exploration program is recommended pointed toward building up how Industry 4.0 may encourage more precise, high quality, constant ecological administration bookkeeping and outside natural detailing in important areas, organization sizes, across various administration jobs and synergistic settings, just as in inventory and worth chains [8]. Ways are plot in which bookkeepers, proficient affiliations and government can help catch the advantages of Industry 4.0 for ecological bookkeeping [8]. These remember instruction and preparing openings and working for proficiency and cheaper ideas which are a vital piece of Industry 4.0 [8]. This imaginative paper gives initial investigation into the capability of current and expected huge ventures all through the world in Industry 4.0 to give an establishment to upgrades in corporate maintainability through more noteworthy take up of natural bookkeeping [8].

TABLE 1: COMPARISION OF LITERATURE REVIEW

\begin{tabular}{|c|c|c|c|c|}
\hline $\begin{array}{l}\text { NAME OF } \\
\text { AUTHOR }\end{array}$ & YEAR & TECHNIQUE & BENEFITS & DRAWBACKS \\
\hline Yajun Zhang & 2020 & $\begin{array}{l}\text { Wireless Sensor } \\
\text { Networks }\end{array}$ & $\begin{array}{l}\text { High-density } \\
\text { transmission and booking } \\
\text { technique in wireless sensor } \\
\text { networks dependent on Wi-Fi }\end{array}$ & $\begin{array}{l}\text { Can be improved more for various } \\
\text { kinds of high density data } \\
\text { transmissions. }\end{array}$ \\
\hline Ling Zhang & 2020 & $\begin{array}{l}\text { neighborhood } \\
\text { Interference } \\
\text { Detection }\end{array}$ & $\begin{array}{l}\text { A nature of framework } \\
\text { steering dependent on } \\
\text { neighborhood impedance } \\
\text { location and moderation in } \\
\text { wireless sensor network }\end{array}$ & $\begin{array}{l}\text { This usage should be loosened up } \\
\text { further to analyze diverse high } \\
\text { speed applications. }\end{array}$ \\
\hline $\begin{array}{l}\text { Xunbao } \\
\text { Wang }\end{array}$ & 2017 & $\begin{array}{l}\text { Medical Internet } \\
\text { of Things }\end{array}$ & $\begin{array}{l}\text { Data Transmission and Access } \\
\text { Protection of Community } \\
\text { Medical Internet of Things }\end{array}$ & $\begin{array}{l}\text { This can be improved more to } \\
\text { discover other sort of high speed } \\
\text { IOT data applications. }\end{array}$ \\
\hline $\begin{array}{l}\text { Wuyungerile } \\
\mathrm{Li}\end{array}$ & 2016 & $\begin{array}{l}\text { Wireless Sensor } \\
\text { Networks }\end{array}$ & $\begin{array}{l}\text { Cascades Partial Aggregation } \\
\text { in Wireless Sensor Networks }\end{array}$ & $\begin{array}{l}\text { There is no much drawback } \\
\text { isolated. This can be improved } \\
\text { more to increment various sorts of } \\
\text { data unwavering quality. }\end{array}$ \\
\hline
\end{tabular}

\section{Conclusion}

In this paper we have reviewed the impact for timely and reliable data transmission in industrial areas. The utilization of data transmission offices speeds up the assortment and spread of data and licenses endorsers with economical terminal gear to appreciate the administrations of huge PC habitats. Nonetheless, existing WSNs are intended to ration energy and not to help timely data transmission. Under high traffic load, the convention conveys data in time utilizing a greatest hub obligation cycle. On account of phone channels, for instance, it utilizes recurrence, stage, and other more intricate sorts of balance, just as different types of sign coding and recoding. This convention is hence an amazingly energy proficient answer for time-basic data delivery. In an inactive organization a greatest hub obligation pattern of just is accomplished. The introduced arrangement utilizes a novel Medium Access Control convention that consolidates geography control components to guarantee timely data delivery and unwavering quality control instruments to manage innately fluctuating wireless connections. Toward the finish of their discussion, or subsequent to trading broadcast messages, the members consent to change to the data transmission mode, and they associate the interchanges channel to the DTD. Various wireless sensor network applications request timely data delivery. This paper reviews how WSNs 
can be dimensioned, conveyed and worked with the end goal that both reliable and timely data delivery is guaranteed while scant energy is saved.

\section{References}

1. Md Abul Kalam Azad 2020 "A two-channel slotted sense multiple access protocol for timely and reliable data transmission in industrial wireless sensor networks" https://doi.org/10.1177/1550147720902007

2. Dash, S., Shakyawar, S.K., Sharma, M. et al. Big data in healthcare: management, analysis and future prospects. J Big Data 6, 54 (2019). https://doi.org/10.1186/s40537-019-0217-0

3. Sinha, A., Kumar, P., Rana, N.P. et al. Impact of internet of things (IoT) in disaster management: a tasktechnology fit perspective. Ann Oper Res 283, 759-794 (2019). https://doi.org/10.1007/s10479-0172658-1

4. Yamila M.Omar 2019 "Business analytics in manufacturing: Current trends, challenges and pathway to market leadership" https://doi.org/10.1016/j.orp.2019.100127

5. By Xue-Ming Yuan 2019 "Impact of Industry 4.0 on Inventory Systems and Optimization" DOI: 10.5772/intechopen.90077

6. Uthayasankar Sivarajah, Muhammad Mustafa Kamal, Zahir Irani, Vishanth Weerakkody, 2017 "Critical analysis of Big Data challenges and analytical methods" https://doi.org/10.1016/j.jbusres.2016.08.001.

7. L. Pradittasnee, S. Camtepe and Y. Tian, "Efficient Route Update and Maintenance for Reliable Routing in Large-Scale Sensor Networks," in IEEE Transactions on Industrial Informatics, vol. 13, no. 1, pp. 144156, Feb. 2017, doi: 10.1109/TII.2016.2569523.

8. Burritt, R., Christ, K. Industry 4.0 and environmental accounting: a new revolution?. AJSSR 1, 23-38 (2016). https://doi.org/10.1186/s41180-016-0007-y

9. Yajun Zhang 2020 "High-density data transmission and scheduling method in wireless sensor networks based on Wi-Fi" https://doi.org/10.1177/1550147720943414

10.Sayyad, S., Mohammed, A., Shaga, V., Kumar, A., \& Vengatesan, K. (2018). Digital Marketing Framework Strategies Through Big Data. In International conference on Computer Networks, Big data and IoT (pp. 1065-1073).

11.Ling Zhang 2020 "A quality of system routing based on neighborhood interference detection and mitigation in wireless sensor network" https://doi.org/10.1177/1550147720968462

12. Wuyungerile Li 2016 "Waterfalls Partial Aggregation in Wireless Sensor Networks" https://doi.org/10.1155/2016/2392149

13.Sayyad, S., Mohammed, A., Shaga, V., Kumar, A., \& Vengatesan, K. (2018). Digital Marketing Framework Strategies Through Big Data. In International conference on Computer Networks, Big data and IoT (pp. 1065-1073)

14. Xunbao Wang 2017 "Data Transmission and Access Protection of Community Medical Internet of Things" https://doi.org/10.1155/2017/7862842 